\title{
Atención del profesorado universitario a estudiantes con necesidades educativas especificas
}

Pedro Ricardo Álvarez-Pérez

Universidad de La Laguna (España) palvarez@ull.es
David López-Aguilar

Universidad de La Laguna (España)

dlopez@ull.es

\section{Resumen}

Esta investigación analiza el rol que desempeña el profesorado en la puesta en práctica de un enfoque inclusivo de la enseñanza y las dificultades que encuentra en su labor educativa y orientadora. Participaron en el estudio una muestra de 111 docentes universitarios, que durante el curso 2012/2013 atendieron a estudiantes con necesidades educativas especificas. Respondieron a un cuestionario estructurado en torno a cuatro dimensiones básicas: 1) información y formación; 2) apoyos y recursos; 3) actuaciones en el aula; 4) actuaciones en las tutorías. Los resultados evidenciaron que el profesorado universitario no está preparado para dar una respuesta educativa adaptada a la diversidad, que demandaba formación en el uso de estrategias y recursos que les permitieran una atención personalizada de todo el alumnado.

\section{Palabras clave}

Enseñanza universitaria, pedagogía universitaria, necesidades educativas especiales, educación inclusiva, Universidad de La Laguna-España. (Fuente: Tesauro de la Unesco).

Recepción: 2014-04-29 | Envío a pares: 2015-01-24 | Aceptación por pares: 2015-07-23 | Aprobación: 2015-08-06 DOI: 10.5294/edu.2015.18.2.1

Para citar este artículo / To reference this article / Para citar este artigo

Álvarez-Pérez, P. R., López-Aguilar, D. (2015). Atención del profesorado universitario a estudiantes con necesidades educativas específicas. Educ. Educ., 18 (2), 193-208. DOI: 10.5294/edu.2015.18.2.1 


\title{
The Attention University Professors Give to Students with Special Educational Needs
}

\begin{abstract}
This research analyzes the role university professors play in implementing an inclusive approach to education and the difficulties they encounter in their work to impart education and guidance. The sample used for the study was comprised of 111 university professors who served students with special educational needs during the 2012/2013 academic year. They answered a questionnaire structured around four basic aspects: 1) information and training; 2) support and resources; 3) classroom performance; and 4) performance in the tutorials. The results showed university professors are not prepared to offer an appropriate response to educational diversity. This necessitates training in the use of strategies and resources that would allow them offer all students personalized attention.
\end{abstract}

\section{Keywords}

University teaching, university education, special educational needs, inclusive education, Universidad de La Laguna, Spain (Source: Unesco Thesaurus). 


\section{Atenção do professorado universitário a estudantes com necessidades educativas especificas}

\section{Resumo}

Esta pesquisa analisa o papel que o professorado desempenha na prática de um enfoque inclusivo do ensino e as dificuldades que encontra em seu trabalho educativo e orientador. Participou do estudo uma amostra de 111 docentes universitários que, durante o curso 2012-2013, atenderam estudantes com necessidades educativas especificas. Responderam a um questionário estruturado em volta de quatro dimensões básicas: 1) informação e formação; 2) apoios e recursos; 3) atuações na sala de aula;4) atuações nas tutorias. Os resultados evidenciaram que o professorado universitário não está preparado para dar uma resposta educativa adaptada à diversidade, que exigia formação no uso de estratégias e recursos que lhe permitissem uma atenção personalizada de todo o alunado.

Palavras chave

Ensino superior, pedagogia universitária, necessidades educativas especiais, educação inclusiva, Universidad de La Laguna - Espanha. (Fonte: Tesauro da Unesco). 


\section{Introducción: la cultura de la igualdad y la inclusión como requisito educativo}

La defensa del principio de igualdad de oportunidades en la sociedad actual ha alcanzado especial relevancia dentro de las políticas sociales de las Naciones Unidas. Desde la Declaración Universal de los Derechos Humanos de 1948, se han ido sucediendo una serie de declaraciones, convenciones y recomendaciones para la lucha contra las discriminaciones de todo tipo y la defensa de la igualdad de oportunidades de todos los colectivos, entre ellos el de las personas con discapacidad. Por ello, la inclusión del alumnado con discapacidad en la educación superior debe plantearse como una exigencia de obligado cumplimiento, de modo que se considere la diversidad como un hecho natural, se prevean las medidas necesarias para el trabajo con grupos heterogéneos y se apliquen las estrategias más adecuadas para que todos aprendan de acuerdo con sus intereses y posibilidades (Eckes y Ochoa, 2005; Blanco, 2008; Infante, 2010; Ocampo, 2013).

La intervención en esta área debe centrarse en el estudiante individualmente y en su progreso, en la compensación de sus dificultades, en la promoción de la accesibilidad, en el fomento de la solidaridad educativa y en el desarrollo de un enfoque interactivo, contextual e inclusivo (Arnaiz, 200o). Es preciso insistir en la necesidad de dejar atrás el planteamiento que colocaba las causas de las limitaciones funcionales en las deficiencias y hacía responsables de su afrontamiento a los propios discapacitados y a sus familiares, para potenciar un enfoque más global, donde se asuma el compromiso social por la mejora de la situación de las personas con necesidades específicas y se fortalezcan las acciones para la igualdad (Alzugaray, Mederos y Sutz, 2011). Los cambios que hagan de la diversidad universitaria una realidad, deberían abarcar la totalidad de los ámbitos de la institución, desde los órganos directivos hasta el mismo entorno del aula, de modo que se hilvanen políticas, normativas, acciones y programas que hagan posible el objetivo de eliminar los obstáculos para conseguir la plena normalización del alumnado con discapacidad. Esto permitiría que este colectivo de estudiantes pudiera cursar los estudios superiores en condiciones de igualdad respecto al resto del alumnado universitario (Alcantud, Ávila y Asensi, 2000; Feliz y Ricoy, 2004).

La educación inclusiva, tan tratada y desarrollada en los niveles básicos de la enseñanza, no ha tenido un desarrollo pleno en la enseñanza universitaria (López, 2005; Álvarez, Alegre, Castro, Castro et al., 2012). Por eso se viene reclamando con insistencia que este enfoque, al igual que en los demás niveles de la enseñanza, se implante en el contexto universitario para dar una verdadera respuesta a la diversidad. El modelo inclusivo en la enseñanza plantea el derecho de todos a una educación de calidad, lo que implica tener en cuenta las necesidades y las potencialidades de cada estudiante. La Unesco (2005, p. 13) se refiere a la educación inclusiva como "un proceso de abordaje y respuesta a la diversidad de las necesidades de todos los alumnos a través de la creciente participación en el aprendizaje, las culturas y las comunidades y de la reducción de la exclusión dentro y desde la educación".

Todos somos diferentes, pero todos debemos convivir juntos y debemos tener la oportunidad de realizar un proyecto formativo que valga para la proyección sociolaboral. En ningún caso, ser diferente debería conducir a un trato desigual y a la falta de igualdad y equidad social. Por eso estamos de acuerdo con que la educación se ejerza sobre los principios de igualdad y equidad, desarrollando un proceso de enseñanza-aprendizaje personalizado e individualizado que promueva una formación inclusiva para todos (Arnaiz, 2000). Esto supone apostar por una actuación orientadora que contribuya al desarrollo de un enfoque inclusivo en la enseñanza universitaria, de tal modo que los estudiantes con discapacidad tengan la formación, la orientación y los apoyos necesarios para que estén en 
condiciones de igualdad y puedan aprovechar las oportunidades de integración social. En definitiva, como señalan Luque y Rodríguez (2005), la formación universitaria debería guiarse desde patrones de aceptación, comprensión y apoyo al estudiante, en términos de accesibilidad, atención a las diferencias, información y ayuda, facilitación de recursos y adaptación, donde se reconozca a cada individuo y sus circunstancias. Compartimos con Bayot, Rincón y Hernández (2002) la idea de que educar en la diversidad supone enseñar en una universidad que incluya a todos y que responda a las características particulares de cada estudiante, impulsando el crecimiento personal y profesional desde sus propias posibilidades y potencialidades.

Para lograr que este enfoque impregne el proceso formativo en la universidad y que se desarrollen buenas prácticas inclusivas (Alonso y Díez, 2008; Izuzquiza, 2012), es necesario que el profesorado adopte una actitud abierta a la innovación, que contemple alternativas pedagógicas adecuadas para un buen trato de las necesidades educativas de todos los estudiantes y que desarrolle sus materias con metodologías adecuadas, para permitir la participación de los estudiantes con discapacidad en igualdad de condiciones que el resto del alumnado (Castro y Alegre, 2009; Voss y Bufkin, 2011). El profesorado que tiene en sus aulas universitarias a estudiantes con necesidades educativas específicas, debe entenderlo como una oportunidad para innovar en su práctica docente y, trabajando en colaboración con otros profesionales, debe encontrar nuevas estrategias de actuación docente que mejoren la calidad de la enseñanza que desarrolla (García y Cotrina, 2011; Leyser, Zeiger y Romi, 2011; Fernández, 2012). Por ello, se requiere un cambio de mentalidad del profesorado y un mejor conocimiento de cuáles son sus dificultades, sus necesidades formativas, los recursos que necesitan, etc., de modo que a partir de la mejora de estas condiciones, se pueda avanzar en la implantación de un enfoque de aprendizaje inclusivo, cooperativo entre todo el alumnado, y de una verdadera formación socializadora.

\section{La función tutorial del profesorado universitario en la atención a la diversidad}

Un plano en el que el profesorado universitario tiene mucho que aportar para lograr una verdadera inclusión del alumnado con necesidades educativas específicas es el de la tutoría académica y personalizada (Zabalza, 2004; Álvarez, 2014). El desarrollo de un sistema de tutorías en la enseñanza universitaria para el alumnado con algún tipo de discapacidad, constituye un factor de calidad que puede contribuir a una mejora sustancial de la integración de todos los estudiantes, la optimización del proceso formativo, la promoción de una mayor igualdad de oportunidades, la potenciación del principio de orientación a lo largo de la vida, el refuerzo de la educación inclusiva, la prevención del abandono de los estudios, etc.

Desde la tutoría universitaria formativa se deben realizar acciones de orientación, seguimiento y asesoramiento para que todos los estudiantes tengan información sobre las características y las opciones de formación universitaria, conozcan la estructura de planes de estudio e itinerarios formativos, resuelvan las dudas y las dificultades de tipo administrativo, conozcan las ayudas y los servicios de que disponen a lo largo de su proceso formativo y desarrollen las competencias necesarias que les permitan competir en igualdad de oportunidades por la inserción laboral (Coriat y Sanz, 2005; García, Asensio, Carballo, García et al., 2005; Álvarez y Lázaro, 2007). La relevancia de la tutoría universitaria ha sido reconocida en el modelo Europeo de Educación Superior, donde el verdadero protagonista es el estudiante, que necesita del profesorado algo más que la enseñanza instructiva presencial del aula (Cano, 2009; Martínez, 2009; Álvarez y López, 2013).

Esta perspectiva de la tutoría universitaria formativa aboga por la atención personalizada del 
alumnado desde el momento en que se incorpora a la educación superior, de modo que se pueda realizar un seguimiento a lo largo de la titulación (Álvarez, 2009; Comes, Parera, Vedriel y Vives, 2011). Se parte del presupuesto de que la tutoría tiene que estar en íntima conexión con el proceso formativo, puesto que en este espacio de engranaje es donde tiene verdadero sentido (Álvarez, Alegre, Castro, Castro et al. 2012; Álvarez, 2013). Podríamos decir que enseñanza y tutoría son las dos caras de la misma moneda: enseñar es ayudar a aprender y para ello se requiere un buen conocimiento del alumnado, sus expectativas, lo que necesita, su motivación, etc. En general, algunas tareas del profesor tutor universitario con estudiantes con discapacidad son las siguientes:

- Ayudar al estudiante a integrarse en la vida universitaria, informándole de los distintos aspectos de la misma y de los recursos a su alcance.

- Atender las necesidades de tipo académico: materiales adaptados, problemas de asistencia a clase, documentos y apuntes, apoyo de los compañeros de clase, etc.

- Informar a los demás profesores, en las comisiones de curso, de las dificultades y necesidades de los estudiantes con discapacidad.

- Facilitar la integración de los estudiantes con discapacidad en la dinámica de las clases, en las tareas de grupo y en las actividades complementarias.

- Proporcionar estrategias para facilitar el estudio y la elaboración de las tareas.

- Motivar al alumnado con discapacidad a lo largo del curso.

- Mediar en la resolución de los problemas que el estudiante con discapacidad pueda tener.
El involucramiento del profesorado en el desarrollo de un modelo de educación inclusivo en la enseñanza universitaria es fundamental; por eso, en este estudio se planteó como objetivo central conocer las dificultades que tenía el profesorado universitario para atender a las necesidades de orientación de los estudiantes con discapacidad. Por tanto, el interrogante en torno al que giró la investigación fue: ¿qué dificultades encuentra el profesorado universitario para atender las demandas educativas y orientadoras planteadas por el alumnado con necesidades educativas específicas?

De manera más concreta, los objetivos que se trazaron en el estudio fueron:

1. Analizar si el profesorado universitario estaba formado para atender a estudiantes con necesidades educativas específicas.

2. Analizar la valoración que hacía el profesorado universitario de los apoyos y recursos con que contaba para atender al alumnado con discapacidad.

3. Conocer el tipo de actuaciones que realizaba el profesorado en el aula para dar respuesta a las necesidades de los estudiantes con discapacidad.

4. Conocer el tipo de intervencionestutoriales que realizaba el profesorado con los estudiantes con discapacidad.

\section{Método}

En correspondencia con los objetivos del estudio, se utilizó una metodología de corte descriptivo/exploratorio no experimental, mediante la aplicación del cuestionario como técnica de recogida de información.

\section{Participantes}

A través del Gabinete de Análisis y Planificación (GAP) de la Universidad de La Laguna, se obtu- 
vieron los datos del total de profesores y profesoras que impartían docencia a estudiantes con discapacidad en alguna de las titulaciones durante el curso académico 2012/2013 ( $n=527$ ). Con esta información, se envió un correo electrónico a la totalidad de docentes identificados con la intención de explicar los objetivos y las finalidades del estudio, así como solicitar su colaboración en la cumplimentación de un cuestionario online.

Del total de profesores identificados, se contó finalmente con la colaboración de 111 participantes, lo que permitió trabajar con una muestra representativa de la población objeto de estudio. El procedimiento de muestreo utilizado aseguró un nivel de confianza del 90\% y un margen de error del 6,95\% de los datos recogidos. La edad media del profesorado se situó en 47,7 años, en un rango que oscilaba entre los 34 y 65 años. En cuanto al género, 58 eran mujeres $(52,22 \%)$ y 53 hombres (47,74\%). En relación con la categoría profesional, el mayor porcentaje correspondió a "profesor titular universitario" $(40,5 \%)$, seguido por el "contratado doctor tipo I" $(24,3 \%)$ (tabla 1).

\section{Tabla 1. Categoría profesional de la población objeto de estudio}

\begin{tabular}{|l|c|}
\hline & Frecuencia/porcentaje \\
\hline Catedrático/a & $8(7,2 \%)$ \\
\hline Profesor Titular Universitario & $45(40,5 \%)$ \\
\hline Titular de Escuela & $14(12,6 \%)$ \\
\hline Contratado Doctor Tipo I & $27(24,3 \%)$ \\
\hline Asociado & $9(8,1 \%)$ \\
\hline Contratado & $5(4,5 \%)$ \\
\hline Ayudante doctor & $2(1,8 \%)$ \\
\hline
\end{tabular}

\section{Instrumentos y procedimiento}

Para la selección del instrumento de recogida de datos se valoraron distintas posibilidades, teniendo en cuenta las características de la muestra a la que iba dirigido y los objetivos que se pretendían alcanzar a través de este estudio. Finalmente, se decidió utilizar un cuestionario on-line, de modo que los participantes pudieran responderlo de manera individual y remitirlo de forma electrónica. Con esta modalidad utilizada para la recogida de datos se garantizaba, además, el anonimato de la respuesta y la confidencialidad de la información recogida. Esta estrategia se consideró adecuada debido a que muchos profesores disponían de poco tiempo para cumplimentar la prueba, por lo que se pensó que este formato podría facilitar tanto la administración como la recogida de datos del estudio.

La validación y fiabilidad de la prueba se realizó a través de diferentes procedimientos que permitieron garantizar la adecuación entre las variables por estudiar y los objetivos de la investigación (McMillan y Shcumacher, 2005). Dado que se trataba de una herramienta on-line, se solicitó la colaboración de expertos en tecnologías de la información y la comunicación $(\mathrm{n}=3)$ con el fin de valorar distintos aspectos relacionados con la plataforma virtual que se iba a emplear, el procedimiento de respuesta, la exportación de datos, etc. Tras la valoración de distintas aplicaciones de encuestas on-line, se decidió utilizar la herramienta Limesurvey, una aplicación de código abierto de encuestas en línea, escrita en lenguaje PHP y bajo el soporte de una base de datos MySOL. Los criterios que se tuvieron en cuenta para la elección de esta plataforma fueron: permitía almacenar un número ilimitado de respuestas de diferentes usuarios; se podían introducir diferentes tipos de preguntas (escalas tipo Likert, preguntas abiertas y cerradas, items de opción múltiple, etc.) y exportaba de manera automática los datos a ficheros compatibles con software estadístico como el Statistical Package for the Sociale Sciences o el Microsoft Excel.

La versión inicial del cuestionario fue sometida a diferentes procedimientos de fiabilidad y validación (McMillan y Shcumacher, 2005). Concretamente, se realizó una prueba piloto en la que se valoraron distintos aspectos relacionados 
con el contenido, la redacción, la comprensión de las preguntas, las opciones de respuesta, etc. Para ello, se aplicó de manera presencial esta primera versión del instrumento a una muestra de personas ( $\mathrm{n}=$ 7) que reunían similares características a las de la población objeto de estudio. Durante el desarrollo de la prueba, tres miembros del equipo de investigación tomaron nota de todas las incidencias relacionadas con la comprensión de los conceptos, la claridad de los enunciados, las alternativas de respuesta, etc.

Del mismo modo, un grupo de profesores universitarios experto en el campo de la inclusión y la integración de estudiantes con discapacidad ( $n=4)$ participó en una prueba de contenido del instrumento. Los criterios que tuvieron en cuenta en el proceso de evaluación del cuestionario fueron: la estructura, la pertinencia, la claridad, la adecuación de las preguntas, etc. Finalmente, expertos en metodología de la investigación $(\mathrm{n}=3$ ) valoraron aspectos referidos a la forma del cuestionario (tipos de preguntas, opciones de respuesta, análisis estadísticos por realizar, etc.).

A partir de todos estos procesos de revisión, se incorporaron diferentes tipos de mejora al instrumento definitivo: se añadieron dos nuevos items, se incorporaron alternativas de respuestas en algunos de ellos, se modificaron enunciados de preguntas y se eliminaron tres preguntas por no tener correspondencia con los objetivos del estudio. La prueba definitiva quedó configurada por un total de 42 preguntas distribuidas en escalas de valoración, preguntas abiertas, ítems dicotómicos y cuestiones de opción múltiple. Las dimensiones que conformaron el instrumento definitivo y que pretendían dar respuesta a los objetivos de la investigación fueron:

1. Datos de identificación (I). Se recogían variables de identificación del profesorado universitario participante en el estudio: sexo, edad, categoría profesional, área de conocimiento, años de experiencia docente y facultad o escuela a la que pertenecía.
2. Formación en atención a la diversidad (II). Este bloque de preguntas ahondaba en la información y la formación que tenía el profesorado universitario para atender a estudiantes universitarios con necesidades educativas específicas.

3. Apoyos y recursos (III). En esta dimensión, se abordaba la información relacionada con los apoyos y recursos con los que contaba el profesorado universitario para ofrecer una atención personalizada a los estudiantes con necesidades educativas específicas.

4. Actuaciones en el aula (IV). En este apartado se valoraban las diferentes intervenciones que realizaba el profesorado universitario en el aula para dar respuesta a las necesidades educativas específicas del alumnado.

5. Actuaciones en las tutorias (V). Finalmente, en esta dimensión se preguntaba por las estrategias y herramientas que utilizaba el profesorado universitario en el espacio de las tutorías para ofrecer una respuesta a las demandas formativas del alumnado con necesidades educativas específicas.

El instrumento fue administrado durante los meses de enero-abril del 2013 al profesorado universitario que durante el curso 2012/2013 atendió a estudiantes con necesidades educativas específicas en sus aulas. El procedimiento que se siguió para administrar el cuestionario fue el envío de un correo electrónico en el que se adjuntaba un hipervínculo con el cuestionario online, una carta en la que se indicaban los objetivos del estudio y se informaba sobre el anonimato de las respuestas, y un documento en el que se solicitaba su libre consentimiento de participación.

\section{Fiabilidad}

El coeficiente alfa de Cronbach se obtuvo mediante el paquete estadístico Statistical 
Package for the Social Sciences (SPSS) en su versión 17.0 para el sistema operativo Microsoft Windows 7. El índice general obtenido para las escalas de valoración recogidas en el cuestionario fue $\alpha=0,873$, lo que indica una adecuada fiabilidad para la medición de las variables objeto de estudio (George y Mallery, 2003).

\section{Análisis de datos}

La información recogida a través del cuestionario on-line fue tratada estadísticamente con el software informático SPSS 17.0 para el entorno Microsoft Windows 7. Concretamente, los análisis que se realizaron fueron:

- Análisis de fiabilidad. Se aplicó un análisis de fiabilidad a los ítems de valoración del cuestionario mediante la prueba alfa de Cronbach, y se valoró a partir de los criterios aportados por George y Mallery (2003).

- Análisis descriptivo (Etxeberria y Tejedor, 2005). Los estadísticos de la media aritmética y la desviación típica fueron calculados para identificar las características principales de la muestra objeto de estudio. Además, se realizó una distribución de frecuencias para cada una de las dimensiones y variables estudiadas con el cuestionario.

- Análisis comparativo entre variables (Rodríguez y Mora, 2011). Se realizaron análisis de contingencia para identificar diferencias estadísticamente significativas entre variables cualitativas.

- Análisis de contenido (López, 2002). Se llevó a cabo un análisis de contenido de las preguntas abiertas del cuestionario, identificando cada una de las aportaciones de los participantes con la codificación $P n$ (donde $P$ hacía referencia a la palabra "profesor" y n representaba el "número" de encuestado).

\section{Resultados}

Los resultados se presentan agrupados en función de las diferentes dimensiones relacionadas con los objetivos y las variables estudiadas.

\section{Formación en atención a la diversidad}

En esta primera dimensión se recogen los datos referidos a la formación que tenía el profesorado universitario para atender a las demandas educativas de los estudiantes con necesidades educativas específicas. El profesorado encuestado manifestó que tenía poca información sobre la respuesta educativa a la diversidad en el contexto de la enseñanza universitaria (93,7\%). Igualmente, señaló que no tenía conocimiento sobre el uso de metodologías concretas (como adaptaciones curriculares, modificaciones de evaluación, etc.) empleadas en los procesos de aprendizaje de los estudiantes con necesidades educativas específicas $(89,2 \%)$.Enestesentido, los docentes eran conscientes de las carencias formativas que tenían. Por ello, plantearon la necesidad de adquirir mejor formación que los capacitara para dar una adecuada respuesta a las demandas educativas de los estudiantes con dificultades formativas. Concretamente, solicitaron formación teórica (19,8\%), formación práctica en metodologías y estrategias de enseñanza $(64,9 \%)$, formación en el diseño y la adaptación de materiales educativos (50,5\%), capacitación en el uso de medios y recursos específicos (45,5\%), en el conocimiento de sistemas alternativos de comunicación (39,6\%) y en la aplicación de estrategias de atención personalizada (49,6\%).

Se encontraron, no obstante, diferencias significativas en función de la categoría profesional del profesorado ( $p$ asociada a $\chi^{2}=0,021$ ). Los profesores ayudantes doctores (50,0\%), asociados $(55,5 \%)$ y titulares de escuela $(28,6 \%)$ manifestaron tener un mayor nivel de conocimiento y formación para atender a las necesidades formativas de estos estudiantes. Igualmente, aquellos vinculados a áreas de conocimiento relacionadas con ciencias sociales y jurídicas tenían una mayor formación 
ISSN 0123-1294 | Educ.Educ. Vol. 18. No. 2 | Mayo-Agosto de 2015 | pp. 193-208.

Universidad de La Sabana | Facultad de Educación

para atender a las necesidades de los estudiantes con algún tipo de discapacidad ( $p$ asociada a $\chi^{2}=$ o,046) (tabla 2).

\section{Apoyos y recursos}

Uno de los aspectos básicos para la atención al estudiantado con necesidades educativas específicas era la disponibilidad de recursos y servicios. En este sentido, el 84,7\% del profesorado indicó que no conocía la existencia de ningún servicio o recurso de apoyo para atender las dificultades de estos estudiantes. Sin embargo, se constataron diferencias estadísticamente significativas ( $p$ asociada a $\chi^{2}=$ 0,000 ) en relación con la experiencia profesional de los encuestados. Concretamente, los docentes universitarios que mayor número de años llevaban impartiendo docencia, manifestaron tener un conocimiento más amplio de los distintos servicios y recursos que ofrecía la institución (figura 1).

Según el 70,3\% del profesorado, esta situación se debía principalmente a que la Universidad de La Laguna no se preocupaba lo suficiente por atender a los estudiantes con necesidades educativas específicas. Además, y desde la perspectiva de los encuestados, la institución universitaria no contaba con los recursos adecuados (82,0\%) para ofrecer una formación de calidad a este tipo de alumnado.

Para dar respuesta a esta realidad, el 76,6\% del profesorado planteó que era necesario mejorar las condiciones de aprendizaje de los estudiantes con discapacidad en la enseñanza universitaria, mediante la introducción de diferentes estrategias como "reducir la ratio profesor/alumno" ( $\left.\mathrm{P}_{1}\right)$; "realizar tutorías especializadas" (P14); "realizar cursos de formación específicos" (P32); "contar con un servicio de asesoramiento específico de apoyo a la discapacidad para el alumnado y el profesorado" (P58); "disponer de personal de apoyo especializado (orientadores, intérpretes, etc.)" (P72); o "eliminar barreras arquitectónicas" (Р93).

\section{Actuaciones en el aula}

La falta de información y formación que tenía el profesorado universitario respecto al proceso de

Tabla 2. Percepción que tiene el profesorado para la atención a estudiantes con discapacidad en función de su categoría profesional

\begin{tabular}{|c|c|c|c|c|c|c|c|}
\hline & $\mathrm{Chi}^{2}$ & $\begin{array}{c}\text { Nada } \\
\text { preparado }\end{array}$ & $\begin{array}{c}\text { Poco } \\
\text { preparado }\end{array}$ & $\begin{array}{l}\text { Nada/poco } \\
\text { preparado }\end{array}$ & $\begin{array}{l}\text { Bastante } \\
\text { preparado }\end{array}$ & $\begin{array}{c}\text { Muy } \\
\text { preparado }\end{array}$ & $\begin{array}{c}\text { Bastante/ } \\
\text { muy } \\
\text { preparado }\end{array}$ \\
\hline Catedrático & \multirow{7}{*}{0,021} & $\begin{array}{c}1 \\
(12,5 \%)\end{array}$ & $\begin{array}{c}7 \\
(87,5 \%)\end{array}$ & $\begin{array}{c}8 \\
(100,0 \%)\end{array}$ & $\begin{array}{c}0 \\
(0,0 \%) \\
\end{array}$ & $\begin{array}{c}0 \\
(0,0 \%)\end{array}$ & $\begin{array}{c}0 \\
(0,0 \%)\end{array}$ \\
\hline $\begin{array}{c}\text { Profesor Titular } \\
\text { Universitario }\end{array}$ & & $\begin{array}{c}3 \\
(6,7 \%)\end{array}$ & $\begin{array}{c}38 \\
(84,4 \%)\end{array}$ & $\begin{array}{c}41 \\
(91,1 \%)\end{array}$ & $\begin{array}{c}4 \\
(8,9 \%)\end{array}$ & $\begin{array}{c}0 \\
(0,0 \%)\end{array}$ & $\begin{array}{c}4 \\
(8,9 \%)\end{array}$ \\
\hline Titular de Escuela & & $\begin{array}{c}1 \\
(7,1 \%)\end{array}$ & $\begin{array}{c}9 \\
(64,3 \%)\end{array}$ & $\begin{array}{c}10 \\
(71,4 \%)\end{array}$ & $\begin{array}{c}4 \\
(28,6 \%)\end{array}$ & $\begin{array}{c}0 \\
(0,0 \%)\end{array}$ & $\begin{array}{c}4 \\
(28,6 \%)\end{array}$ \\
\hline $\begin{array}{c}\text { Contratado Doctor } \\
\text { Tipo } 1\end{array}$ & & $\begin{array}{c}3 \\
(11,1 \%)\end{array}$ & $\begin{array}{c}20 \\
(74,1 \%)\end{array}$ & $\begin{array}{c}23 \\
(85,2 \%)\end{array}$ & $\begin{array}{c}4 \\
(14,8 \%)\end{array}$ & $\begin{array}{c}0 \\
(0,0 \%) \\
\end{array}$ & $\begin{array}{c}4 \\
(14,8 \%)\end{array}$ \\
\hline Asociado & & $\begin{array}{c}1 \\
(11,1 \%)\end{array}$ & $\begin{array}{c}3 \\
(33,3 \%)\end{array}$ & $\begin{array}{c}14 \\
(44,4 \%)\end{array}$ & $\begin{array}{c}4 \\
(44,4 \%)\end{array}$ & $\begin{array}{c}1 \\
(11,1 \%)\end{array}$ & $\begin{array}{c}5 \\
(55,5 \%)\end{array}$ \\
\hline Contratado & & $\begin{array}{c}2 \\
(40,0 \%)\end{array}$ & $\begin{array}{c}3 \\
(60,0 \%)\end{array}$ & $\begin{array}{c}5 \\
(100,0 \%)\end{array}$ & $\begin{array}{c}0 \\
(0,0 \%)\end{array}$ & $\begin{array}{c}0 \\
(0,0 \%)\end{array}$ & $\begin{array}{c}0 \\
(0,0 \%)\end{array}$ \\
\hline Ayudante Doctor & & $\begin{array}{c}0 \\
(0,0 \%)\end{array}$ & $\begin{array}{c}1 \\
(50,0 \%)\end{array}$ & $\begin{array}{c}1 \\
(50,0 \%)\end{array}$ & $\begin{array}{c}1 \\
(50,0 \%)\end{array}$ & $\begin{array}{c}0 \\
(0,0 \%)\end{array}$ & $\begin{array}{c}1 \\
(50,0 \%)\end{array}$ \\
\hline
\end{tabular}


Figura 1. Apoyos y recursos de la ULL

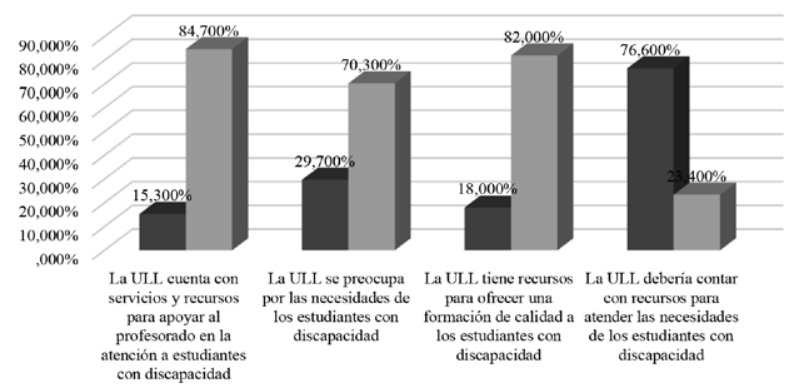

aprendizaje de los estudiantes con discapacidad dificultó la atención específica que debían ofrecer para responder a sus necesidades formativas. Entre los principales problemas que encontró el profesorado se destacó la falta de materiales y programas concretos (47,5\%), la escasez de sistemas alternativos de comunicación (37,5\%) y la configuración de espacios adecuados para la enseñanza (30,0\%) (figura 2).

Pese a estos obstáculos, la mayor parte del profesorado manifestó haber realizado algún tipo de adaptación curricular con la intención de dar respuesta a las necesidades de los estudiantes con dificultades formativas. Concretamente, realizaron modificaciones en las que se llevó a cabo una atención más personalizada e individualizada (50,0\%), adaptaron la evaluación a las necesidades de cada estudiante (32,5\%), modificaron la metodología docente $(22,5 \%)$ y usaron material de clase adaptado para que el alumnado pudiera acceder a los contenidos (20,0\%). Sin embargo, el $47,5 \%$ indicó que no había empleado técnicas concretas en el aula para facilitar la participación e integración de los estudiantes con discapacidad en la enseñanza universitaria.

\section{Actuaciones en las tutorias}

En este último apartado, se recogían las distintas medidas de atención y orientación personalizada que el profesorado universitario

\section{Figura 2. Dificultades para atender al alumnado con discapacidad}

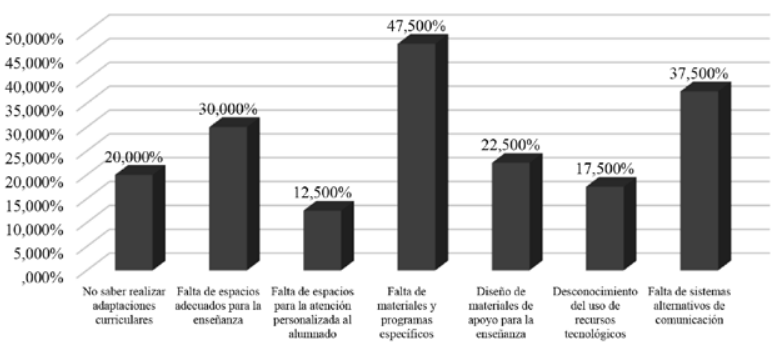

utilizaba en las tutorías para ofrecer una respuesta adaptada a las necesidades formativas de los estudiantes con dificultades educativas. En este sentido, los docentes encuestados indicaron que era importante que en la enseñanza universitaria existiera un Plan de Tutorías específicas para los estudiantes con necesidades educativas, con la finalidad de facilitarles los procesos de adaptación e integración a la enseñanza universitaria (65,0\%). No obstante, el 80,0\% del personal docente señaló que no había desarrollado ningún tipo de actuación personalizada e individualizada en tutorías para el alumnado con estas características, ni tampoco había desarrollado estrategias específicas para impulsar la asistencia de estos estudiantes a las tutorías (87,5\%).

No obstante, una parte del profesorado planteó que había utilizado técnicas y estrategias específicas en el espacio de la tutoría para facilitar el acceso al contenido de las diferentes materias (22,5\%). Concretamente, el profesorado indicó que había hecho uso de "fichas visuales (adaptación del lenguaje, gráficos, esquemas, etc.)" (P4); "explicaciones al intérprete de signos previas al inicio de las clases" (P27); "atenciones personalizadas a través de la web" (P42); "comunicaciones orales más despacio" (P58); o "trabajos con maquetas y representaciones gráficas” (81).

El $82,5 \%$ del profesorado universitario señaló que no realizaba ningún tipo de seguimiento de 
los estudiantes con discapacidad que no acudían a clase. Además, un alto porcentaje $(85,0 \%)$ indicó que tampoco empleaban estrategias específicas para que los compañeros de clase ayudaran a estos estudiantes que no podían acudir regularmente al aula por motivos derivados de su situación personal.

A pesar de estos datos, un 55,0\% consideró que la actuación con el alumnado con discapacidad en las tutorías era la adecuada, aunque también señalaron que este recurso era poco utilizado por este colectivo de estudiantes (57,5\%). Además, el profesorado universitario indicó que el uso que le daban los estudiantes con necesidades educativas a las tutorías era principalmente para la resolución y aclaración de dudas académicas (70,0\%), para tratar aspectos personales $(32,5 \%)$ o a fin de solicitar la "adaptación de materiales para las clases" (P92). En este sentido, los profesores encuestados solicitaron estrategias y herramientas para ofrecer un mayor apoyo y una mejor atención personalizada al alumnado con necesidades educativas específicas desde el espacio de la tutoría. Concretamente, demandaron "mayor apoyo institucional" (P31); "creación de un servicio específico de discapacidad para el asesoramiento al profesorado y al alumnado" (P42); "disminuir la ratio alumno/profesor" (P68); etc.

\section{Discusión}

En este estudio sobre las dificultades que encuentra el profesorado universitario para atender al alumnado con necesidades educativas específicas y contribuir a su proceso de integración al contexto de la educación superior y al espacio del aula, se han puesto de relieve los problemas y las carencias formativas que tienen los docentes para dar una respuesta educativa adaptada a la diversidad. Los resultados evidencian que queda todavía mucho terreno por recorrer para que el profesorado universitario se incorpore a esa visión de la enseñanza para todos y a ese modelo inclusivo que defienden Stainback, Stainback y Jones (1999) o Ainscow (2001). Si se pretende que la igualdad de oportunidades y la normalización penetren en las aulas universitarias y se extiendan más allá de acciones e intervenciones concretas y específicas, es preciso incidir y mejorar la preparación y participación del profesorado en este modelo de universidad inclusiva. Coincidimos con González (2008) cuando señala que una de las barreras para la atención a la diversidad es la inadecuada formación inicial del profesorado. En el caso de la educación superior, la formación de los docentes está orientada básicamente a la especialización curricular, siendo inexistente o quedando en un segundo plano la preparación para dar respuesta a otros problemas de la enseñanza, como puede ser la atención a las necesidades educativas específicas del alumnado. Por eso coincidimos con Bayot, Rincón y Hernández (2002, p. 71) cuando afirman que "un alumno con problemas no implica nuevas áreas de trabajo, únicamente exige movilizar la creatividad del profesional para la búsqueda de nuevas respuestas ante demandas propias de su responsabilidad". La realidad es que el profesorado universitario no ha sido preparado para responder a las necesidades individuales del alumnado con discapacidad y la acción educativa se plantea más hacia la uniformidad que a tener en cuenta las diferencias. Blanco (2008) resalta la necesidad de que las actividades educativas estén adaptadas a las características de todo el alumnado, dado que esto queda en entredicho debido a que los docentes no han sido formados para llevar a cabo este proceso e introducir las modificaciones necesarias a fin de dar una respuesta ajustada a la diversidad del alumnado del aula. Como ha quedado reflejado, solo una parte de los docentes encuestados se ha preocupado por atender las necesidades educativas del alumnado con discapacidad y ha llevado a cabo en sus aulas adaptaciones del currículo. Las adaptaciones curriculares realizadas las han planteado a partir de las demandas hechas por el propio alumnado con discapacidad, y tienen que ver con la atención personalizada (50\%), la evaluación (32,5\%), la metodología (22,5\%) y los materiales 
de enseñanza (22,5\%). En el otro extremo se sitúa el profesorado (un 17\%) que no llevó a cabo ningún tipo de adaptación curricular, por no disponer de formación o por falta de recursos para poder realizarla.

Los datos presentados en el estudio reflejan claramente cuál es la percepción de los docentes sobre la formación de sus compañeros y de la suya propia para atender al alumnado con discapacidad: salvo un porcentaje minoritario ( $16 \%$ de la muestra), la mayoría opinaron que estaban poco o nada preparados, y que el nivel de formación de que disponían lo consideraban como regular o malo. Se destaca, sin embargo, que el profesorado con mayor categoría profesional manifestara sentirse menos preparado para atender los estudiantes con necesidades educativas específicas, frente al profesorado novel que consideraba estar más formado para afrontar esta tarea educativa. Encontramos también que aquellos docentes adscritos a áreas del conocimiento del ámbito social, humanístico y jurídico señalaron estar mejor capacitados en el tema de atención a la diversidad que los docentes que pertenecen a áreas más técnicas.

Tal como apuntan Opertti y Brady (2011), la mejora de la capacitación de los docentes constituye uno de los requisitos de la universidad actual para la implantación de un modelo de educación inclusiva, y una condición ineludible para que los docentes puedan dar una respuesta individualizada y adecuada a las necesidades del alumnado con discapacidad. Las demandas formativas que señalan para atender de manera adecuada al alumnado con discapacidad tienen que ver con la formación en estrategias de enseñanza, metodologías de adaptación curricular, uso de materiales didácticos, herramientas para la atención personalizada y conocimiento de sistemas alternativos de comunicación.

Si se logra mejorar la formación de los docentes en este ámbito, podrían participar en el desarrollo de medidas adecuadas de adaptación de la enseñanza, creando entornos de aprendizaje más inclusivos para el desarrollo de una atención más personalizada del alumnado, o la puesta en práctica de guías y programas de orientación específica. Esto permitiría, como señalan Luque y Rodríguez (2008), el desarrollo de una enseñanza más normalizada y adaptada a las necesidades de todo el alumnado universitario, para que la cultura de la igualdad llegara a todos, y para que cada individuo dispusiera de la posibilidad de definir y fortalecer su identidad personal, social y profesional.

Respecto a las medidas de apoyo y seguimiento, muchos docentes consideraron que sería necesario disponer de un programa específico de tutorías para este alumnado, que hiciera posible una respuesta más concreta e individualizada. Asimismo, reclamaron una mejor coordinación entre los servicios universitarios que participan y atienden al alumnado con necesidades educativas específicas. Coincidimos con Muntaner (2010) cuando insiste en la importancia de que las administraciones educativas estén comprometidas con el modelo inclusivo y de que el profesorado cuente con los apoyos necesarios para trasladarlo a la práctica. La diversidad de servicios existentes, y la falta de coordinación entre ellos, genera muchas veces dificultades, no solo a los profesionales, sino también al alumnado con discapacidad que recurre a ellos.

\section{Conclusiones}

El análisis realizado en torno a la participación del profesorado en el desarrollo de un modelo de enseñanza inclusiva, nos lleva a las siguientes conclusiones:

El profesorado universitario considera que se debería optimizar la formación para dar una mejor respuesta adaptada a las necesidades específicas del alumnado con discapacidad.

Las principales demandas de formación que plantean los docentes tienen que ver con el conocimiento de metodologías para realizar adaptaciones curriculares, el uso de estrategias de enseñanza y el manejo de recursos didácticos. 
ISSN 0123-1294 | Educ.Educ. Vol. 18. No. 2 | Mayo-Agosto de 2015 | pp. 193-208.

Universidad de La Sabana | Facultad de Educación

Los docentes tienen dificultades para llevar a cabo una atención personalizada del alumnado con necesidades educativas específicas dado que no dominan sistemas alternativos de comunicación.
El profesorado considera que se debería implantar en la enseñanza universitaria un sistema de tutoría personalizada para atender a los estudiantes con necesidades educativas específicas.

\section{Referencias}

Ainscow, M. (2001). Desarrollo de escuelas inclusivas. Ideas, propuestas y experiencias para mejorar las instituciones escolares. Madrid: Narcea.

Alcantud, F., Ávila, V.y Asensi, M. (2000). La integración de estudiantes con discapacidad en los estudios superiores. Universidad de Valencia: Servicio de publicaciones.

Alonso, A. y Díez, E. (2008). Universidad y discapacidad: indicadores de buenas prácticas. Siglo Cero. Revista Española sobre Discapacidad Intelectual, 39 (2), 82-98.

Álvarez, P. (2002). La función tutorial en la Universidad: una apuesta por la mejora de la calidad de la enseñanza. Madrid: EOS.

Álvarez, P. (2009). Tutoría para la integración del alumnado con discapacidad: un reto para la universidad actual. En Castro, J. y Alegre, O. (coords.). Alumnado con discapacidad en la universidad. Tenerife:Turquesa.

Álvarez, P., Alegre, O. M., Castro, J. F., Castro, F. M., Sanabria, A. et al. (2012). Tutoría universitaria inclusiva: Guía de buenas prácticas para la orientación de estudiantes con necesidades educativas especificas. Madrid: Narcea.

Álvarez, P. (2013). La Tutoría como eje articulador del proceso de aprendizaje del alumnado universitario. Revista Qurriculum, 26, 73-88.

Álvarez, P. y López, D. (2013). La tutoría académica personalizada para estudiantes universitarios deportistas de alto nivel. Revista Brasileña de Orientación Profesional, 14 (2), 239-253.

Álvarez, P. (2014). La función tutorial del profesorado universitario: una competencia de la labor docente en el contexto del EEES. Revista Portuguesa de Pedagogía, 47 (2), 85-106.

Álvarez, V.y Lázaro, A. (2002). Calidad de las universidades y orientación universitaria. Málaga: Aljibe.

Alzugaray, S., Mederos, L. y Sutz, J. (2011). La investigación científica contribuyendo a la inclusión social. Revista CTS: Revista Iberoamericana de Ciencia, Tecnología y Sociedad, 17 (6), 11-30.

Arnaiz, P. (2000). Educar en y para la diversidad. En Soto, F. y Navarro, J. Nuevas tecnologías, viejas esperanzas: las nuevas tecnologías en el ámbito de la discapacidad y las necesidades especiales. Murcia: Consejería de Educación y Cultura. 
Bayot, A., Rincón, B. y Hernández, F. (2002). Orientación y atención a la diversidad: descripción de programas y acciones en algunos grupos emergentes. Revista Electrónica de Investigación y Evaluación Educativa, 8 (1).

Blanco, R. (2008). Marco conceptual sobre la educación inclusiva. En La educación inclusiva: el camino hacia el futuro. Aportes a las discusiones de los talleres de la Conferencia Internacional de Educación. Unesco: Oficina Internacional de Educación.

Cano, R. (2009). Tutoría universitaria y aprendizaje por competencias. ¿Cómo lograrlo? Revista Interuniversitaria de Formación del Profesorado, 12 (1), 181-204.

Castro, J. y Alegre, O. (coords.) (2009). Alumnado con discapacidad en la Universidad. Guías para el profesorado. Tenerife: Turquesa.

Comes, G., Parera, B., Vedriel, G. y Vives, M. (2011). La inclusión del alumnado con discapacidad en la universidad: la opinión del profesorado. Innovación Educativa, 21, 173-183.

Coriat, M. y Sanz, R. (2005). Orientación y tutoría en la Universidad de Granada. Granada: Editorial Universidad de Granada.

Eckes, S.y Ochoa, T. (2005). Students with disabilities: transition from high school to higher education. American Secondary Education, 33 (3), 1-16.

Etxeberria, J.y Tejedor, F.J. (2005). Análisis descriptivo de datos en educación. Madrid: La Muralla.

Feliz, T.y Ricoy, M. (2004). Aproximación del alumnado con necesidades educativas especiales en la Universidad. Revista Española de Orientación y Psicopedagogía, 15, (1), 155-168.

Fernández, J. M. (2012). Capacidades y competencias docentes para la inclusión del alumnado en la educación superior. Revista de la Educación Superior, XLI (2), 9-24.

García, N., Asensio, I., Carballo, R., García, M. y Guardia, S. (2005). La Tutoría Universitaria ante el proceso de armonización europea. Revista de Educación, 337, 89-210.

García, M.y Cotrina, M. (2011). Accesibilidad, inclusión e innovación docente en la Universidad. Quaderns digitals: Revista de Nuevas Tecnologías y Sociedad, 67, 1-12.

George, D. y Mallery, P. (2003). SPSS for Windows step by step: a simple guide and reference. 11.o Update. Boston: Allyn \& Bacon.

González, M. (2008). Diversidad e inclusión educativa: algunas reflexiones sobre el liderazgo en el centro escolar. Revista Iberoamericana de Calidad, Eficacia y Cambio en Educación, 6 (2).

Infante,M. (2010). Desafíos a la formación docente: inclusión educativa. Proyecto Fondecyt. Estudios Pedagógicos, XXXVI (1).

Izuzquiza, D. (2012). El valor de la inclusión educativa de jóvenes con discapacidad intelectual en las instituciones de Educación Superior: el programa Promentor. Bordón, 64 (1), 109-125. 
ISSN 0123-1294 | Educ.Educ. Vol. 18. No. 2 | Mayo-Agosto de 2015 | pp. 193-208.

Universidad de La Sabana | Facultad de Educación

Leyser,Y.,Zeiger,T.y Romi,S. (2011). Changes in Self-efficacy of Prospective Special and General Education Teachers: Implication for inclusive education. International Journal of Disability, Development and Education, 58 (3), 241-255.

López, F. (2002). El análisis de contenido como método de investigación. XXI Revista de Educación, 4, 167-179.

López, M. (2005). Educación, amor, ética... caminos para construir un sueño: la escuela inclusiva. Tavira, 21, 11-27.

Luque, D. y Rodríguez, G. (2005). Accesibilidad y adaptaciones curriculares al alumnado con discapacidad en la Universidad. Una reflexión docente. I Congreso Nacional de Universidad y Discapacidad, Salamanca.

Luque, D. y Rodríguez, G. (2008). Alumnado universitario con discapacidad: elementos para la reflexión psicopedagógica. Revista Española de Orientación y Psicopedagogía, 19 (3), 270-281.

Martínez, M. (2009). La orientación y la tutoría en la universidad en el marco del Espacio Europeo de Educación Superior (EEES). Revista Fuentes, 9, 78-97.

McMillan, J. H. y Schumacer, S. (2005). Investigación educativa. Una introducción conceptual. Madrid: Pearson Addison Wesley.

Muntaner, J. (2010). De la integración a la inclusión: un nuevo modelo educativo. En Arnaiz, P., Hurtado, M. y Soto, F. (coord.). Veinticinco años de integración escolar en España: tecnología e inclusión en el ámbito educativo, laboral y comunitario. Murcia: Consejería de Educación, Formación y Empleo.

Ocampo, A. (2013). Inclusión de estudiantes en situación de discapacidad a la educación superior. Desafíos y oportunidades. Revista Latinoamericana de Educación Inclusiva, 6 (2), 227-239.

Opertti, R. y Brady, J. (2011). Developing inclusive teachers from an inclusive curricular perspective. Prospects, 41 (3), 459-472.

Rodríguez, M. y Mora, R. (2011). Análisis de tablas de contingencia. En Rodríguez, M. J. y Mora, R. Estadistica informática: casos y ejemplos con el SPSS. Alicante: Servicio de Publicaciones de la Universidad de Alicante.

Stainback, S., Stainback, W. y Jones, J. (1999). Aulas Inclusivas. Madrid: Narcea.

Unesco (2005). Guidelines for Inclusión: Ensuring Access to Education for All. Paris: Unesco.

Voss, J. A. y Bufkin, L. J. (2011). Teaching All Children: Preparing Early Childhood Preservice Teachers in Inclusive Settings. Journal of Early Childhood Teacher Education, 32 (4), 338-354.

Zabalza, M. (2004). Aprovechamiento didáctico de la tutoría: estrategias y técnicas de la acción tutorial. I Jornadas sobre la tutoría en la enseñanza universitaria. Salamanca. 